\title{
Bidirectional-pumped tunable fiber laser using a voltage-controlled Fabry-Perot Etalon filter
}

\author{
Anir Syazwan Sharbirin*, Muhamad Zharif Samion, Aisah Muhamad, Mohammad Faizal Ismail, \\ Harith Ahmad
}

Photonics Research Center, University of Malaya, 50603 Kuala Lumpur, Malaysia

*Corresponding author: harith@um.edu.my

\section{Article history}

Received 18 February 2017

Accepted 9 August 2017

\begin{abstract}
Fabry-Perot based filters have long been an attractive interferometric device due to its simple construction and versatility for various applications. In this paper, we demonstrate a bidirectionalpumped tunable Thulium-doped Fiber Laser (TDFL) using a voltage-controlled Fabry-Perot Etalon (FPE) filter. The filter allows a maximum tuning range of $\sim 42.0 \mathrm{~nm}$, starting from $1965.40 \mathrm{~nm}$ up to $2007.35 \mathrm{~nm}$. The generated laser also exhibits a relatively low threshold power of $\sim 62.57 \mathrm{~mW}$ and narrow bandwidth of $\sim 0.16 \mathrm{~nm}$. The tuning capability exhibits an exponential growth relationship which can be exploited for precise automatic electronic control.
\end{abstract}

Keywords: Tunable laser; bidirectional-pump; thulium-doped fiber laser; Fabry-Perot Etalon filter

\section{INTRODUCTION}

In recent years, lasers in the two-micron region have gained popularity as an interesting research subject, mainly due to its ability to strongly absorb water [1] and carbon dioxide gas [2] more than the other common near infrared fiber lasers. It is also well known to be an eye-safe laser [3] because of its water-absorbing property which makes it a potential candidate for precise biomedical surgery especially for laser eye surgery [4]. Since two-micron lasers have such high absorption coefficient for carbon dioxide gas added with being an 'eye-safe' laser, it can be exploited for use in Light Detection and Ranging (LIDAR) [5] and can also be used in the atmospheric related study [2].

Two-micron fiber laser can be generated from Thulium- or Holmium-doped fiber. However, a study by Wu et al. have reported that Thulium-based lasers have an advantage in terms of its quantum efficiency which is close to $200 \%$ [6]. This is actually the result of the cross relaxation energy transfers of $\mathrm{Tm}^{3+}$ ions from the ${ }^{3} \mathrm{H}_{6}$ and ${ }^{3} \mathrm{H}_{4}$ levels to the ${ }^{3} \mathrm{~F}_{4}$ level. Thulium-doped fibers (TDF) also have a substantially better slope efficiency than other rare earth doped fibers as it can overcome the Stokes limit [7, 8]. Therefore, Thulium-doped fiber laser (TDFL) is highly sought out as an efficient two-micron laser source. A significant improvement to the TDFL can be accomplished by incorporating a wavelength tuning device into the laser setup. Tunable TDFL has numerous application in sensing [5, 9-11] and spectroscopy [12-14].

The simplest setup for a tunable laser employs grating based filter such as arrayed waveguide gratings (AWG) [15] and tunable bandpass filter (TBF) [16]. However, AWG and TBF is expensive and is not easily available for the two-micron wavelength region if compared with the other wavelength region, such as C-band. An electro-tunable laser is also more of an interest as it is possible to control it through direct electronic circuitry. In this paper, a bidirectional-pumped voltagecontrolled tunable TDFL is demonstrated by utilizing a Fabry-Perot
Etalon (FPE) filter as the wavelength selective mechanism. FPE is an interferometer based filter and this type of filter had already been demonstrated as a reliable wavelength tuning device with wide tuning range [17-19]. Bidirectional pumping scheme is chosen for the laser setup in order to produce high pumping power and achieve high gain $[20,21]$. The proposed laser has promising potential to be applied in sensing and spectroscopy.

\section{EXPERIMENTAL SETUP}

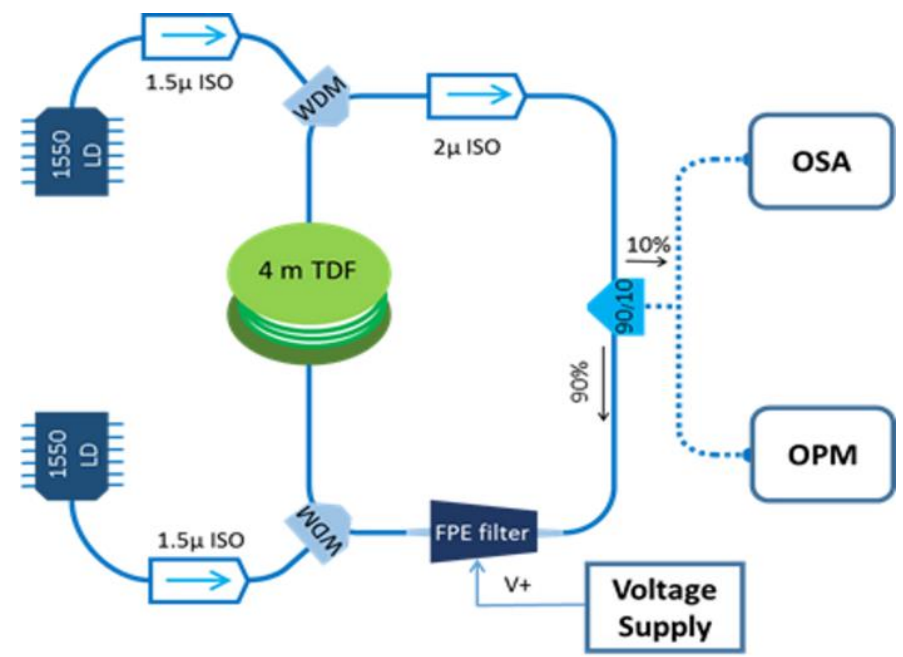

Fig. 1 Schematic diagram of the Tunable TDFL cavity.

The setup for the voltage-controlled TDFL is described in Fig. 1. Two PSL-450 $1550 \mathrm{~nm}$ laser diodes by Princeton Lightwave are used as the input pump source with a total combined power of $457 \mathrm{~mW}$. Each 
LD is connected to an optical isolator to prevent backscattering, then spliced to the $1550 \mathrm{~nm}$ port of a 1550/2000 wavelength division multiplexer (WDM). The $2000 \mathrm{~nm}$ port of the WDM is spliced to a 4-m-long Thulium-doped fiber (TDF) provided by OFS, which has a measured absorption coefficient of $\sim 20 \mathrm{dBm}^{-1}$ at $1550 \mathrm{~nm}$ and a core diameter of $5 \mu \mathrm{m}$. Before the ring cavity is completed, the bidirectionally pumped TDF is tested for its gain, noise figure (NF) and power scaling capability. A two-micron laser with a center wavelength of $1934.8 \mathrm{~nm}$ is used as the signal into the cavity. The signal is amplified in forward, backward and bidirectional configuration while their respective gain, NF and power scaling slope efficiency are compared in Fig. 2(a) and (b).

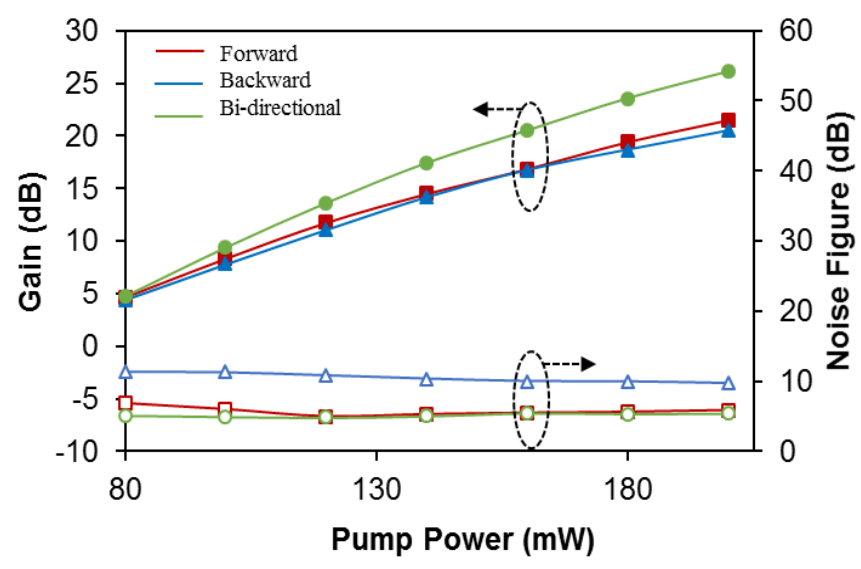

(a)

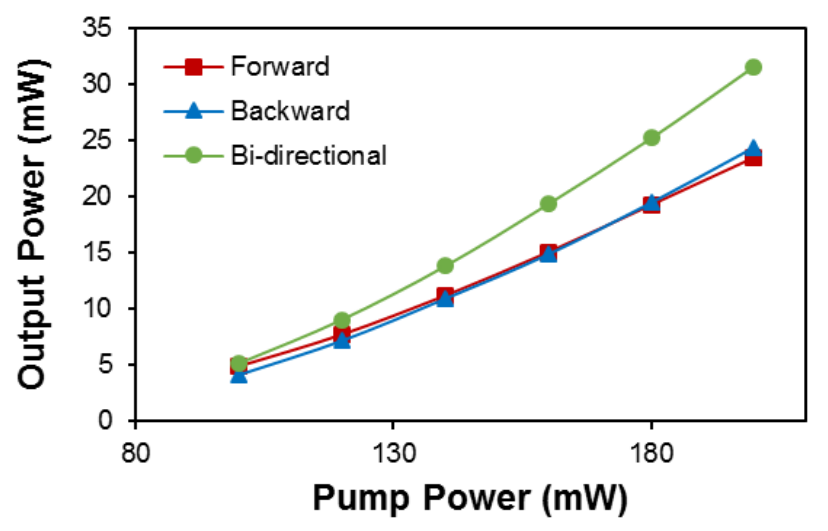

(b)

Fig. 2 Comparison of (a) Gain and NF and (b) power scaling at different configuration.

The ring cavity is then completed by inserting a 90/10 coupler that is connected after a $2000 \mathrm{~nm}$ isolator which ensures unidirectional propagation of light in the cavity. The $90 \%$ output of the coupler passes through an FPE filter (Model no.: TB2500M-Z26) which is connected to the $2000 \mathrm{~nm}$ port of another WDM to complete the cavity. The FPE filter is usually constructed by using two parallel reflecting surfaces (mirrors) that are separated by a certain distance from one another. Multiple and successive reflections of light occur between the mirrors and produce an interference fringe output by constructive and destructive interference. The distance between the internal mirrors in the FPE filter can cause a phase shift at the output. The FPE filter selected has a piezoelectric transducer which moves the mirrors position and is controlled by using a conventional power supply, which provides a stable DC voltage output, accurate up to $0.01 \mathrm{~V}$. By changing the voltage input to the FPE filter, the wavelength of the laser can be tuned, following the phase shift of the FPE filter. The FPE filter is provided by JDS Uniphase and has an insertion loss of $\sim 3.12 \mathrm{~dB}$ measured at $1998.0 \mathrm{~nm}$. The filter has a bandwidth (BW) of 0.4 to 0.9 $\mathrm{nm}$, free spectral range (FSR) of 50.0 to $85.0 \mathrm{~nm}$ and finnese of 90 to $130 \mathrm{~nm}$. It is also noted that the FPE filter used is designed for lasers operating at $1550 \mathrm{~nm}$ wavelength. Regardless, since it works based on interference, the filter should theoretically be able to function with the two-micron wavelength region provided that the loss by the internal mirrors are minimal.

Finally, the $10 \%$ output of the laser is extracted for measurement of the optical spectrum and the optical power. The output is analyzed using a Yokogawa AQ6375 optical spectrum analyzer (OSA) with a spectral resolution of $0.05 \mathrm{~nm}$ and an S302C THORLABS Optical Power Meter (OPM).

\section{RESULTS AND DISCUSSION}

Based on Fig. 2(a), the gain of bidirectional pumping is the highest with a slope of $0.18 \mathrm{~dB} / \mathrm{mW}$, while for forward pumping and backward pumping is $0.13 \mathrm{~dB} / \mathrm{mW}$. On the other hand, no clear difference is seen for the NF between the bidirectional and forward pumping setup. Similar results are attained in a previous analysis by Shukla et al. [21], and it is inferred that gain difference between the three configurations is due to the saturating effect of amplified spontaneous emission (ASE). Bidirectional pumping allows the saturation by ASE on both ends of the fiber, thus, allowing higher gain. However, this does little improvement on the noise figure of the setup. Meanwhile, the power scaling capability is shown in Fig. 2(b) whereby the bidirectional setup shows a significant improvement owing to its gain capability. The bidirectional pump allows a slope efficiency of $27 \%$ compared to 18 $20 \%$ for forward and backward pump. Clearly, bi-directional pumping scheme is the better option in terms of gain and power scaling

By completing the ring cavity, light starts to oscillate strongly and stimulated emission takes over. The constructed Thulium-doped fiber laser (TDFL) starts to lase at a threshold input power of $38.41 \mathrm{~mW}$ and a peak wavelength of $1998.85 \mathrm{~nm}$. The generated laser has a high sidemode suppression ratio (SMSR) of $\sim 57.03 \mathrm{~dB}$ and a 3 -dB bandwidth of $\sim 0.34 \mathrm{~nm}$, as displayed in Fig. 3. By incorporating a Fabry-Perot Etalon (FPE) filter into the cavity setup (as described in Fig. 1), the laser can easily be tuned by varying the input voltage to the FPE filter. The full tunable spectrum is displayed in a three-dimensional (3D) plot as shown in Fig. 4. The configuration has a maximum tuning range of $\sim 42.0 \mathrm{~nm}$, with wavelength peaks at $1965.40 \mathrm{~nm}, 1970.90 \mathrm{~nm}, 1975.65$ nm, $1981.22 \mathrm{~nm}, 1988.03 \mathrm{~nm}, 1992.41 \mathrm{~nm}, 1998.91 \mathrm{~nm}$ and 2007.35 $\mathrm{nm}$ at input voltages of $3.48 \mathrm{~V}, 7.57 \mathrm{~V}, 9.74 \mathrm{~V}, 10.43 \mathrm{~V}, 12.06 \mathrm{~V}, 13.08$ $\mathrm{V}, 13.83 \mathrm{~V}$ and $14.96 \mathrm{~V}$ respectively. At $1975.65 \mathrm{~nm}$, the laser has the highest SMSR, with a value of $\sim 43.21 \mathrm{~dB}$, which indicates that the FPE filter is optimum at that wavelength. Interestingly, the FPE filter produces a narrower laser, at an average 3 -dB bandwidth value of 0.16 $\mathrm{nm}$, in comparison to the initial spectrum.

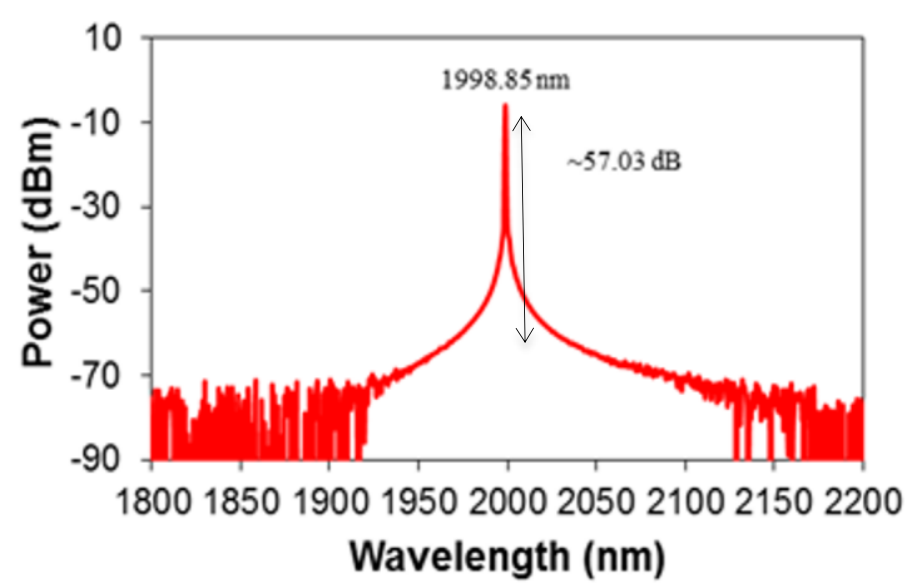

Fig. 3 The laser spectrum before incorporating the FPE filter.

The threshold of the laser with and without the FPE filter is compared in Fig. 5. The lasing threshold is higher after the FPE filter is inserted at an input power of $\sim 62.57 \mathrm{~mW}$, meanwhile, the laser efficiency also drops from $8.36 \%$ to $6.13 \%$. These difference in the values can be associated with the loss introduced by the FPE filter. In comparison to a work reported by Wei at. al., the laser produced by our 
FPE filter is much sharper with a significantly lower lasing threshold and better laser efficiency since we use a smaller input pumping power to produce the same amount of output power [17]. In comparison to his work, our laser setup is far better in terms of laser quality i.e. efficiency, threshold, and bandwidth, attributed to the bidirectional-pumping configuration and also to the FPE filter used.

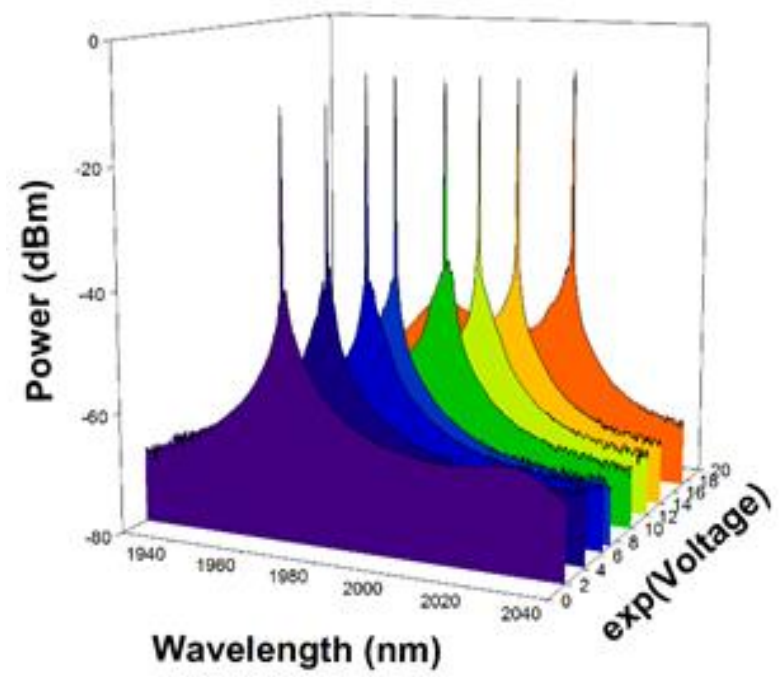

Fig. 4. The 3D-plot of the tunable spectrum after incorporating the FPE filter.

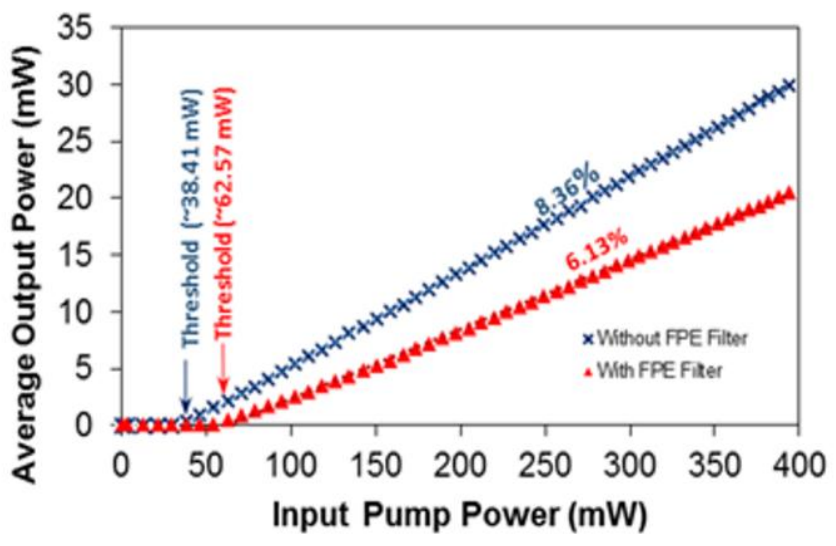

Fig. 5. Comparison of the laser efficiency before and after using the FPE filter.

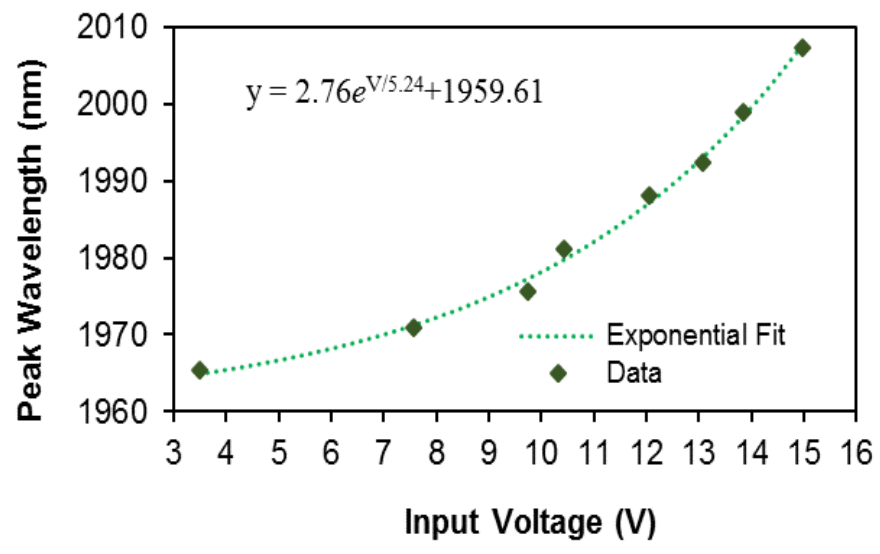

Fig. 6 Exponential curve fit of the peak wavelength versus input voltage.

To further study our tunable TDFL setup, the relationship of the tuned peak wavelengths is plotted against the voltage, as described in Fig 6. The input voltage with the peak wavelength exhibits a good exponential curve fitting with an $\mathrm{R}^{2}$ value of 0.9953 , which is near to 1. The equation of the curve is

$$
y=2.76 e^{V / 5.24}+1959.61
$$

whereby, $\mathrm{y}$ is the peak wavelength and $\mathrm{V}$ is the input voltage.

This tunable laser setup has a potential to be further improved since it is voltage-controlled. Equation (1) can be used to calibrate the electronics to control the voltage with the output wavelength, thus, the wavelength of the laser should be able to be tuned directly by using simple electronic circuitry.

\section{CONCLUSION}

In summary, an efficient tunable TDFL using a voltage-controlled FPE filter has been demonstrated. The bidirectional-pumping configuration allows a higher pumping input power for better laser operation. The tunable TDFL has a maximum range of $\sim 42.0 \mathrm{~nm}$ reaching a laser peak of $2007.35 \mathrm{~nm}$. The laser produced by the FPE filter has a narrow bandwidth of $\sim 0.16 \mathrm{~nm}$ and a relatively low lasing threshold of $\sim 62.57 \mathrm{~mW}$ with good laser efficiency. The wavelength tunability via voltage control also exhibited an exponential trend indicating a possibility as a promising candidate to be utilized as a tunable laser source for spectroscopy and sensing applications.

\section{ACKNOWLEDGEMENT}

This work was financially supported by the University of Malaya under the Research University Grant and Ministry of Higher Education Malaysia (Grant No,: ROGS BR003-2016).

\section{REFERENCES}

[1] J. A. Curcio and C. C. Petty, "The near infrared absorption spectrum of liquid water," Journal of the Optical Society of America, vol. 41, pp. $302-$ 304, 1951.

[2] E. F. Barker, "Carbon dioxide absorption in the near infra-red," The Astrophysical Journal, vol. 55, p. 391, 1922.

[3] A. N. S. Institute, American National Standard for Safe Use of Lasers, ANSI Z136.1-2007, Orlando, Florida: Laser Institute of America, 2007.

[4] S. L. Karsten Scholle, Philipp Koopmann, Peter Fuhrberg, "2 $\mu \mathrm{m}$ laser sources and their possible applications," Frontiers in Guided Wave Optics and Optoelectronics. vol. 1, B. Pal (Ed.), Germany: InTech, 2010, pp. 471500. Available from: https://www.intechopen.com/ books/ frontiers-inguided-wave-optics-and-optoelectronics/2-m-laser-sources-and-theirpossible-applications

[5] K. S. Repasky, S. Humphries, and J. L. Carlsten, "Differential absorption measurements of carbon dioxide using a temperature tunable distributed feedback diode laser," Review of Scientific Instruments, vol. 77, p. 113107, 2006.

[6] J. Wu, S. Jiang, T. Luo, J. Geng, N. Peyghambarian, and N. P. Barnes, "Efficient thulium-doped 2- $\mu \mathrm{m}$ germanate fiber laser," IEEE Photonics Technology Letters, vol. 18, pp. 334-336, 2006.

[7] S. D. Jackson and S. Mossman, "Efficiency dependence on the $\mathrm{Tm}^{3+}$ and $\mathrm{Al}^{3+}$ concentrations for $\mathrm{Tm}^{3+}$-doped silica double-clad fiber lasers," Applied Optics, vol. 42, pp. 2702-2707, 2003.

[8] D. Shen, J. Mackenzie, J. Sahu, W. Clarkson, and S. Jackson, "High-power and ultra-efficient operation of a $\mathrm{Tm}^{3+}$-doped silica fiber laser," Advanced Solid-State Photonics, 2005, p. 516.

[9] U. N. Singh, J. Yu, M. Petros, T. Refaat, and K. Reithmaier, "Development of a pulsed 2-micron integrated path differential absorption lidar for $\mathrm{CO}_{2}$ measurement," SPIE Optical Engineering + Applications, , pp. 887209887209-8, 2013.

[10] B. Yun, Y. Wang, A. Li, and Y. Cui, "Tunable fiber laser based fiber Bragg grating strain sensor demodulation system with enhanced resolution by digital signal processing," Microwave and Optical Technology Letters, vol. 48, pp. 1391-1393, 2006.

[11] M. Li, K. Liu, W. Jing, and G.-D. Peng, "Fiber ring laser intra-cavity absorption spectroscopy for gas sensing: Analysis and experiment," Journal of the Optical Society of Korea, vol. 14, pp. 14-21, 2010. 
[12] B. G. Lee, M. A. Belkin, R. Audet, J. MacArthur, L. Diehl, C. Pflügl, et al., "Widely tunable single-mode quantum cascade laser source for midinfrared spectroscopy," Applied Physics Letters, vol. 91, p. 231101, 2007.

[13] H. Y. Ryu, W.-K. Lee, H. S. Moon, and H. S. Suh, "Tunable erbium-doped fiber ring laser for applications of infrared absorption spectroscopy," Optics Communications, vol. 275, pp. 379-384, 2007.

[14] S. Zheng, Y. Zhu, and S. Krishnaswamy, "Tunable fiber ring laser absorption spectroscopic sensors for gas detection," Procedings SPIE 8692, Sensors and Smart Structures Technologies for Civil, Mechanical, and Aerospace Systems, 10 - 14 March 2013, San Diego, California, United States. pp. 869213-869213-9, 2013.

[15] M. J. R. Heck, A. L. Porta, X. J. M. Leijtens, L. M. Augustin, T. d. Vries, B. Smalbrugge, et al., "Monolithic AWG-based Discretely Tunable Laser Diode With Nanosecond Switching Speed," IEEE Photonics Technology Letters, vol. 21, pp. 905-907, 2009.

[16] Z. Sun, D. Popa, T. Hasan, F. Torrisi, F. Wang, E. J. Kelleher, et al., "A stable, wideband tunable, near transform-limited, graphene-mode-locked, ultrafast laser," Nano Research, vol. 3, pp. 653-660, 2010.
[17] Y. Wei, K. Hu, B. Sun, and T. Wang, "All-fiber widely wavelengthtunable thulium-doped fiber ring laser incorporating a Fabry-Perot filter," Laser Physics, vol. 22, pp. 770-773, 2012.

[18] C.-S. Kim, F. N. Farokhrooz, and J. U. Kang, "Electro-optic wavelengthtunable fiber ring laser based on cascaded composite Sagnac loop filters," Optics Letters, vol. 29, pp. 1677-1679, 2004.

[19] A.-P. Luo, Z.-C. Luo, and W.-C. Xu, "Tunable and switchable multiwavelength erbium-doped fiber ring laser based on a modified dualpass Mach-Zehnder interferometer," Optics Letters, vol. 34, pp. 2135 2137, 2009 .

[20] Z. Li, A. M. Heidt, N. Simakov, Y. Jung, J. M. O. Daniel, S. U. Alam, et al., "Diode-pumped wideband thulium-doped fiber amplifiers for optical communications in the 1800 - $2050 \mathrm{~nm}$ window," Optics Express, vol. 21, pp. 26450-26455, 2013.

[21] P. Shukla and K. P. Kaur, "Performance Analysis of EDFA for different pumping configurations at high data rate," Global Journal of Researches in Engineering-F: Electrical and Electronic Engineering, vol. 13, pp. 1924, 2013. 